\title{
On Balancing between Minimum Energy and Minimum Delay with Radio Diversity for Wireless Sensor Networks
}

\author{
Sofiane Moad, Morten Tranberg Hansen, Raja Jurdak, Branislav Kusy, Nizar Bouabdallah, Adlen Ksentini \\ Email: Sofiane.Moad@inria.fr, mth@cs.au.dk, Raja.Jurdak@csiro.au \\ Brano.Kusy@csiro.au, Nizar.Bouabdallah@inria.fr, Adlen.Ksentini@irisa.fr
}

\begin{abstract}
The expected number of transmissions (ETX) metric represents the link quality in wireless sensor networks, which is highly variable for a specific radio and it can influence dramatically both of the delay and the energy. To adapt to these fluctuations, radio diversity has been recently introduced to improve the delivery rate but at the cost of increases in energy for wireless sensor networks. In this paper, we propose a scheme for radio diversity that can balance, depending on the traffic nature in the network, between minimizing the energy consumption or minimizing the end-to-end delay. The proposed scheme combines the benefit of two metrics, which aim separately to minimize the energy consumption, and to minimize delay when delivering packets to the end-user. We show by both analysis and simulation that our proposed scheme can adapt to the type of traffic that can occur in a network so that it minimizes both energy and delay for the respective traffic classes.
\end{abstract}

Keywords: Wireless Sensor Networks (WSNs), Radio, link quality, QoS.

\section{INTRODUCTION}

WSNs refer to a broad class of wireless networks consisting of small, inexpensive and energy limited devices. In these types of networks, sensor nodes have the responsibility of collecting data and communicating them to a collecting point, called the Base Station (BS). Most sensor nodes have limited power and energy, which requires them to balance between lifetime and application-specific performance.

Radio diversity becomes a promising solution for balancing between lifetime/application-specific performances. Specifically, in [1], the authors explored radio diversity in order to improve the reliability at the expense of an increase of energy consumption. In their solution, a radio with a better quality, which may be costly in energy, is chosen within a sensor node in order to transmit packets. However, it may exist cases where this choice is not necessary. For example, this is the case of specific routed packets that do not have a sense of urgency. Indeed the non-urgent packet do not need to be forwarded with a better radio quality, as it is costly in energy. We deal with such a situation in this paper. We explore the energy/delay trade-off with radio diversity, depending on the type of the traffic in the network. Specifically, we consider urgent and normal packets where urgent packets need to be delivered as fast as possible to the end-user, while normal packets do not and therefore are delivered with a minimum energy. In multiple radios, we show that referring only to the choice of link quality, is an appropriate metric to minimize the delivery delay requirements. However, to deliver a packet with minimum energy, it is required to take into account the energy cost of transmitting and receiving through a specific link. In our previous works regarding radio diversity, we focused in [2] on how to route packets with an energy efficient way, while in [3] we focused on how to balance energy consumption through the network to extend network lifetime. However, we did not give a packet prioritization when routing. We propose, in this paper, a scheme where a node can balance between energy and delay targets depending on a traffic class.

The rest of the paper is organized as follows. Section II presents the related work. Section III presents the system model, while Section IV shows the proposed strategy. Section VI evaluates the performance of the proposed strategy before we conclude in Section VII.

\section{RELATED WORK}

Multi-radio systems have been intensively studied in recent year due to their ability to increase the performance of a network [4]. The use of multiple radios in data communication systems is a common technique refereed to as Multiple Input Multiple Output (MIMO). We refer in the following some of recent works related to LAN for MIMO.

In [5], a Weighted Cumulative Expected Transmission Time $(W C E T T)$ is proposed for wireless mesh routers with multiple radio interfaces. WCETT is used to measure the quality of a path and it is based on the Expected Transmission Time (ETT) metric, which is essentially the expected time to transmit a packet of a certain size over a link. WCETT combines the Summation of ETT (SETT) over a path, with Bottleneck Group ETT (BGETT), which is the sum of expected transmission time of a bottleneck channel. Depending on the parameter set for SETT and BGETT, WCETT aims to achieve a good trade-off between delay and throughput.

Cooperative or virtual MIMO scheme is used in WSN applications, in which nodes group together to form a virtual antenna arrays and transmit the data cooperatively. An example of such schemes is discussed in [6] and [7]. They improve the reliability of links by employing antenna diversity with specific algorithms for their selection. 
Our proposed scheme differs from the prior approaches, as we consider diversity in link quality to decide which radio link that would be used to forward data using a routing protocol.

Recently, the authors in [1] proposed a multi-radio scheme for WSNs. They explored the diversity in ETX [8] metric present in each radio in order to improve the reliability performance at the cost of increase in energy.

In our previous works regarding radio diversity, specifically in [2], we focused on how to route packets with an energy efficient way, and in [3] we focused on how to balance energy consumption over the network to extend network lifetime. However, the main difference between this work and the ones in [2] and [3] is that we give a packet priority when routing, so that a node can enable ETX [1] and weighted ETX $(W E T X)[2]$ metrics depending on a traffic class.

To our best knowledge, this is the first work that addresses a multi-hop routing in order to balance between minimizing energy and delay for multiple-radio nodes for WSNs.

\section{SYSTEM MODEL}

We assume a WSN consisting of $N$ sensors deployed in a field to continuously monitor an environment. We denote the $i$-th sensor node by $n_{i}$ and the corresponding set of sensor nodes $S=\left\{n_{1}, n_{2}, \ldots, n_{N}\right\}$ where $|S|=N$. We make the following assumptions about sensor nodes and the network:

- Sensor nodes and the BS are all stationary after the deployment.

- Nodes in Single Input Single Output (SISO) are equipped with a single radio $r_{1}$ or $r_{2}$, while nodes in the MIMO are equipped with multiple radios (in our case $r_{1}$ and $r_{2}$ ). We denote $E_{r_{1}}^{t x}, E_{r_{2}}^{t x}$ the energy of transmitting a packet for $r_{1}$ and $r_{2}$, respectively. Similarly, we denote $E_{r_{1}}^{r x}, E_{r_{2}}^{r x}$ the energy of reception a packet by $r_{1}$ and $r_{2}$, respectively.

- We denote the set of $n_{i}$ neighbors by $N e_{i}$. Each node $n_{i}$ can reach its neighbor $n_{j}\left(n_{j} \in N e_{i}\right)$ with $E_{r_{1}}^{t x}$ or $E_{r_{2}}^{t x}$ for $r_{1}$ and $r_{2}$, respectively.

- Links are symmetric [9], i.e., if $n_{i} \in N e_{j}$, then $n_{j} \in$ $N e_{i}$. Links are not perfect and they are characterized by a $P R R$ (packet reception ratio), which reflects the link quality. The $P R R$ is defined as the probability of a packet reception over a link. We assume that the $P R R$ during the deployment is constant. We denote $P R R_{r_{1}}$ and $P R R_{r_{2}}$ the $P R R$ of links for $r_{1}$ and $r_{2}$, respectively. We assume that the $P R R$ of the link is symmetric. If $n_{i}$ have a $P R R$ $P R R_{r_{1}}(l)$ to its neighbor $n_{j} \in N e_{i}$, then $n_{j}$ have also the same $P R R_{r_{1}}(l)$ to its $n_{i} \in N e_{j}$ using $r_{1}$.

- Nodes use a collection tree protocol to send data toward a BS according to some routing metric. The metric in $S I S O$ mode is the ETX $=\frac{1}{P R R}$ metric. The ETX [8] metric represents the expected number of transmissions a node needs in order to successfully deliver a packet. It is to be noted that the state-of-the-art collection tree protocol (CTP) [10], [11] uses ET X to forward data.

- Nodes use infinite retransmissions to improve their packet delivery rate to the BS.
The objective of our proposed protocol is to adapt to the applications that require two type of packets (urgent and normal packets). Therefore, we aim at delivering urgent packets with a minimum delay, while ensuring a minimum energy for delivering normal packets.

\section{Proposed strategy}

In this section, we describe the routing strategies $R_{d}, R_{e}$ and $R_{e+d}$ which aims to minimize delay, minimize energy or balance between minimum energy and minimum delay, respectively.

\section{A. Minimizing the delay with radio diversity}

Having multiple radios at a node enables it to choose the radio with lower $E T X$ when forwarding a packet as shown in [1]. By enabling $M I M O$, nodes may avoid retransmissions that cause an overhead in delay. In other words, a node chooses the radio with higher $P R R$ (or lower ETX) in order to avoid retransmissions, and therefore it minimizes the delay of transmitted packets. We call this proposed strategy $R_{d}$, for routing with multiple radios that aims to minimize the delay.

To illustrate the motivation of using $R_{d}$ against using only one radio $\left(R_{r_{1}}\right.$ and $\left.R_{r_{2}}\right)$, let us consider the example presented in Fig. 1.

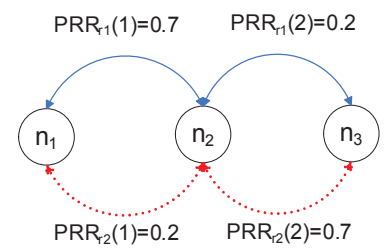

Fig. 1. $P R R$ of the links in both radios $r_{1}$ and $r_{2}$.

Fig. 1 shows the $P R R$ of links for two radios, $r_{1}$ and $r_{2}$. With enabling $R_{d}$, the link of radio $r_{1}$ will be used to communicate between nodes $n_{1}$ and $n_{2}$, while the link $r_{2}$ will be used to communicate between the nodes $n_{2}$ and $n_{3}$. If we consider the delay of transmitting a packet as one time unit (i.e., $d_{t r}=1$ ), and if we consider that the retransmission of the failed packet occurs at $d_{w}=0.5$ time unit after a failed transmission, then the delay for the packet to arrive from $n_{1}$ to $n_{3}$ in $R_{r_{1}}$ and $R_{r_{2}}$ is $T D_{r_{1}}=T D_{r_{2}}=$ $\left(\frac{1}{0.7}+\frac{1}{0.2}\right) \times\left(d_{t r}+d_{w}\right)-2 \times d_{w}=8.64$ time unit, while the corresponding delay in $R_{d}=3.28$ time unit. Here, the delay is definitely better in the $R_{d}$ scheme compared to both $R_{r 1}$ and $R_{r 2}$. The benefit of using the $R_{d}$ scheme becomes more interesting when some of the links in $r_{1}$ is better than $r_{2}$ and vice versa, so that nodes will be able to use the better links. It is to be noted that even though $R_{d}$ minimizes the delay, it is not suitable for WSNs applications where the most important metric is energy consumption.

\section{B. Minimizing the energy with radio diversity}

To minimize the energy consumption when forwarding packets in $M I M O$, we consider the $R_{e}$ scheme. In contrary 
to $R_{d}$ where only link qualities are considered for a packet forwarding, $R_{e}$ considers in addition the transmission and reception energy costs of the link. We call this cost a weighted $E T X$ (or $W E T X$ for short). In other words, $R_{e}$ chooses a minimum $W \operatorname{ETX}(j, k)=E_{r_{i}}^{t x} \times \operatorname{ET} X_{r_{i}}(j, k)+E_{r_{i}}^{r x}, i=1,2$, where $n_{j} \in N e_{k}$, and $(j, k)=(1 . . N, 1 . . N)$. For a link $(i, j)$, $W \operatorname{ETX}(i, j)$ reflects the expected energy consumed over this link. Therefore, $R_{e}$ uses $W E T X$ to first choose the most energy efficient radio and then forward the data towards the BS. Using this, It is important to highlight that $\left(E_{r_{i}}^{t x}, E_{r_{i}}^{r x}\right)$, $i=1,2$ are local information available at a node.

To illustrate the $R_{e}$ protocol operation, we refer to the same example shown in Fig. 1. We consider in this example the $\left(E_{r_{1}}^{t x}, E_{r_{1}}^{r x}\right)=(4,1)$ energy unit, $\left(E_{r_{2}}^{t x}, E_{r_{2}}^{r x}\right)=(1,1)$ energy unit. In the example, for the links $(1,2)$, nodes $n_{1}$ and $n_{2}$ both estimate the cost $\operatorname{WETX}(1,2)=4 / 0.7+1=6.71$ when using the link of $r_{1}$, and the cost $\operatorname{WETX}(1,2)=6$ when using the link of $r_{2}$. The same for the links $(2,3)$, at nodes $n_{2}$ and $n_{3}, W \operatorname{ETX}(2,3)=21$ when choosing the link of $r_{1}, W \operatorname{ETX}(2,3)=2.4$, when choosing the link of $r_{2}$. Therefore, the nodes will choose the link that requires the least energy cost. Consequently, $n_{1}$ and $n_{2}$ choose $r_{2}$ as $6<6.71$, while $n_{2}$ and $n_{3}$ choose $r_{2}$ as $2.4<21$. As a result, the energy gain at the link $(1,2)$ when using $R_{e}$ compared to $R_{d}$ is $1-6 / 6.71=10.58 \%$.

\section{Balancing between minimum energy and minimum delay with radio diversity}

Based on the $R_{d}$ and $R_{e}$ protocols and depending on the type of generated packets, we propose $R_{e+d}$ that combines the $R_{e}$ and $R_{d}$ protocols. We consider that two kinds of packets can occur in the network: urgent and normal packets. Urgent packets occur with the probability $p_{\text {urgent }}$ and normal packets with probability $1-p_{\text {urgent }}$.

The operation of $R_{e+d}$ is as follows. If a packet is urgent, it will be forwarded with the $R_{d}$ scheme until the delivery at $B S$ to minimize the delay, in the contrary, if the packet is normal, it will be forwarded with $R_{e}$ until the delivery at BS to minimize the energy. Many applications may be applicable when using $R_{e+d}$. For example, it can be used in environmental monitoring applications where the sensed data that are not critical can be forwarded with a minimum energy, while those having information of a specific importance (for example in monitoring for fire detection) can be delivered as quick as possible to the end-user.

In Table I, we summarized the name of the different strategies and their meaning.

TABLE I

ROUTING STRATEGIES.

\begin{tabular}{|l|l|}
\hline Name & Meaning \\
\hline$R_{r_{i}}$ & Routing using $S I S O$ on $r_{i}, i=1,2$ \\
\hline$R_{d}$ & Routing with $M I M O$ that ensures a minimum delay \\
\hline$R_{e}$ & Routing with $M I M O$ that ensures a minimum energy \\
\hline$R_{e+d}$ & Routing with $M I M O$ that ensures both minimum \\
\hline
\end{tabular}

\section{ANALYTICAL RESULTS}

To compare between the different routing strategies, we derive an analytical model of the energy consumption and the delay for each strategy. In our analysis we consider a chain or a path of $L$ links rooted at a BS (see Fig. 2). We also consider a free energy consumption at the $\mathrm{BS}$, as it is not energy constrained. We then calculate the energy spent by nodes and the packet delay each level $l(1 \leqslant l \leqslant L)$ of the chain topology, when the node $n_{1}$ is generating packets periodically. It is to be noted that $l$ is the number of the link in the chain and it represents the link between node $n_{l}$ and $n_{l+1}$.

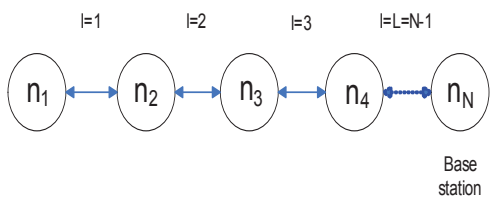

Fig. 2. Chain topology of $L$ links rooted at the BS.

\section{A. Energy model}

We denote $E_{r_{i}}(l), E_{d}(l), E_{e}(l)$ and $E_{d+d}(l)$, the energy consumed at link $l$ for each strategy $R_{r_{i}}, R_{d}$, and $R_{e}, i=1,2$, respectively as follows.

$$
E_{r_{i}}(l)= \begin{cases}\frac{E_{r_{i}}^{t x}}{P R R_{r_{i}}(l)}+E_{r_{i}}^{r x}, & \text { if } l<L \\ \frac{E_{r_{i}}^{t x}}{P R R_{r_{i}}(l)}, & \text { if } l=L\end{cases}
$$

$$
E_{d}(l)= \begin{cases}\frac{E_{r_{1}}^{t x}(l)}{P R R_{r_{1}}(l)}+E_{r_{1}}^{r x}, & \text { if } P R R_{r_{1}}(l)>P R R_{r_{2}}(l) \\ \frac{E_{r_{1}}^{t x}}{P R R_{r_{1}}(l)}, & \text { and if } l<L \\ \frac{E_{r_{2}}^{t x}}{P R R_{r_{2}}(l)}+E_{r_{1}}^{r x}, & \text { if } P R R_{r_{1}}(l)>P R R_{r_{2}}(l) \\ \frac{E_{r_{2}}^{t x}}{P R R_{r_{2}}(l)}, & \text { and if } l<L \\ & \text { if } P R R_{r_{1}}(l) \leq P R R_{r_{2}}(l) \\ & \text { and if } l=L\end{cases}
$$

$$
E_{e}(l)=\left\{\begin{array}{c}
\min \left(\frac{E_{r_{1}}^{t x}}{P R R_{r_{1}}(l)}+E_{r_{1}}^{r x}, \quad \text { if } l<L\right. \\
\left.\frac{E_{r_{2}}^{t x}}{P R R_{r_{2}}(l)}+E_{r_{2}}^{r x}\right) \\
\min \left(\frac{E_{r_{1}}^{t x}}{P R R_{r_{1}}(l)}, \frac{E_{r_{2}}^{t x}}{P R R_{r_{2}}(l)}\right), \text { if } l=L
\end{array}\right.
$$

We then calculate the total energy consumed for each generated packet at the node $n_{1}, T E_{r_{i}}, T E_{d}$ and $T E_{e}$ for each 
strategy $R_{r_{i}}, R_{d}$ and $R_{e}, i=1,2$, respectively as follows.

$$
\begin{aligned}
T E_{r_{i}} & =\sum_{l=1}^{l=L}\left(E_{r_{i}}(l)\right) \\
T E_{d} & =\sum_{l=1}^{l=L}\left(E_{d}(l)\right) \\
T E_{e} & =\sum_{l=1}^{l=L}\left(E_{e}(l)\right)
\end{aligned}
$$

Based on equations (4) and (5), and depending on the type of the generated packets, we calculate the total energy $T E_{e+d}$ of $R_{e+d}$ protocol.

$$
T E_{e+d}= \begin{cases}T E_{d}, & \text { if the packet is urgent } \\ T E_{e}, & \text { otherwise }\end{cases}
$$

\section{B. Delay model}

Similar to the energy model, we denote $D_{r_{i}}(l), D_{d}(l)$, $D_{e}(l)$, and $D_{e+d}(l)$ the delay of transmitting a packet at link $l$ for each strategy $R_{r_{i}}, R_{d}$, and $R_{e}, i=1,2$, respectively as follows.

$$
D_{r_{i}}(l)=\frac{1}{P R R_{r_{i}}(l)} \times\left(d_{t r}+d_{w}\right)-d_{w}
$$

where $d_{t r}$ is the packet delay transfer and $d_{w}$ represents a constant delay that a node will wait before retransmitting a packet when the transmission fails. Note that we set the same packet transfer time for the two radios in order to only focus on the delay caused by the diversity in $P R R$.

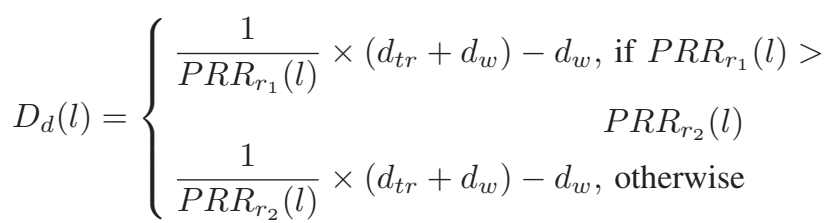

$D_{e}(l)=\left\{\begin{array}{c}\frac{1}{P R R_{r_{1}}(l)} \times\left(d_{t r}+d_{w}\right)-d_{w}, \text { if } E_{r_{1}}^{t x} \times P R R_{r_{1}}(l) \\ \frac{1}{P R R_{r_{2}}(l)} \times\left(d_{t r}+d_{w}\right)-d_{w}, \text { otherwise }\end{array}\right.$

The total delay $T D_{r_{i}}, T D_{e}$ and $T D_{d}$ for $R_{r_{i}}, R_{e}$ and $R_{d}$, $i=1,2$, respectively is calculated as follows.

$$
\begin{aligned}
T D_{r_{i}} & =\sum_{l=1}^{l=L}\left(D_{r_{i}}(l)\right) \\
T D_{d} & =\sum_{l=1}^{l=L}\left(D_{d}(l)\right) \\
T D_{e} & =\sum_{l=1}^{l=L}\left(D_{e}(l)\right)
\end{aligned}
$$

Based on equations (9) and (10), and depending on the type of the generated packets, we calculate the total delay $T D_{e+d}$ of $R_{e+d}$ protocol.

$$
T D_{e+d}= \begin{cases}T D_{d}, & \text { if the packet is urgent } \\ T D_{e}, & \text { otherwise }\end{cases}
$$

Fig. 3 shows the total energy consumed by varying $E_{r_{1}}^{t x}$. In this scenario, we set $p_{\text {urgent }}=0.5$. From Fig. 3 , we observe that by increasing $E_{r_{1}}^{t x}, R_{e}$ converges to use the links with the cheapest radio $r_{2}$ in terms of energy consumption. In addition, we observe that $R_{e+d}$ has an energy consumption in between $R_{e}$ and $R_{d}$, as normal and urgent packets are forwarded with $R_{e}$ and $R_{d}$, respectively. In addition, we observe that $R_{e}$ ensures the lowest total energy consumption compared to the other strategies.

Fig. 4 shows the total end-to-end delay versus $E_{r_{1}}^{t x}$. Similar to the previous scenario, we set $p_{\text {urgent }}=0.5$ and we observe that $R_{d}$ ensures the lowest delay compared to the other strategies. As expected, we observe that $R_{e+d}$ has an end-to-end delay in between $R_{d}$ and $R_{e}$, as $R_{e+d}$ forwards urgent packets with $R_{d}$ and normal packets with $R_{e}$.

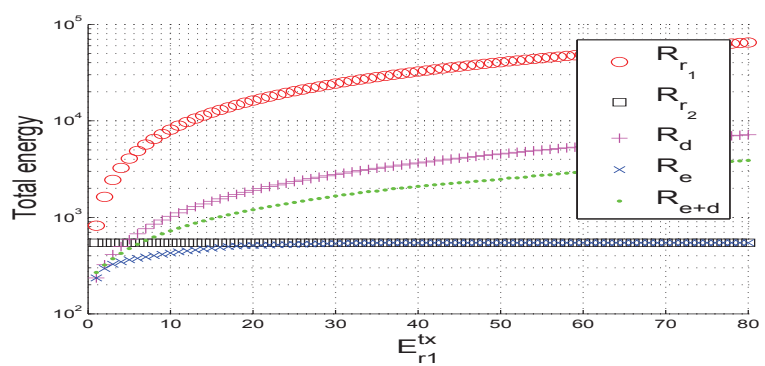

Fig. 3. Total consumed energy with varying $E_{r_{1}}^{t x}$.

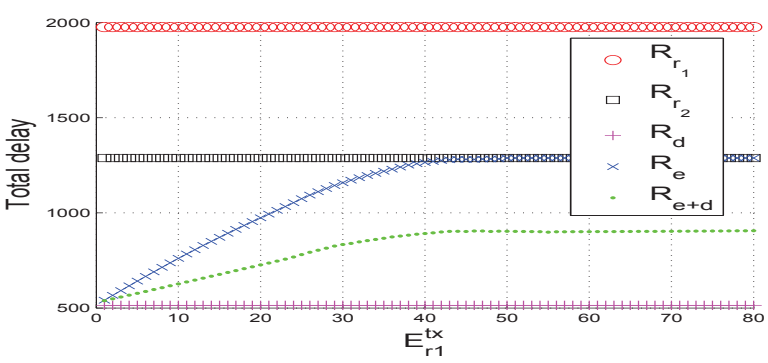

Fig. 4. Total end-to-end delay with varying $E_{r_{1}}^{t x}$.

Fig. 5 shows the total energy consumed with $p_{\text {urgent }}$. In this scenario, we set $E_{r_{1}}^{t x}=10, E_{r_{2}}^{t x}=1$, and $E_{r_{1}}^{r x}=E_{r_{2}}^{r x}=0.1$. We observe from the Fig. 5 that $R_{e+d}$ varies with $p_{\text {urgent }}$, while $R_{d}$ and $R_{e}$ are independent of the type of the generated packets to be forwarded. Indeed, $R_{d}$ and $R_{e}$ aim exclusively to minimize delay or energy, respectively. 


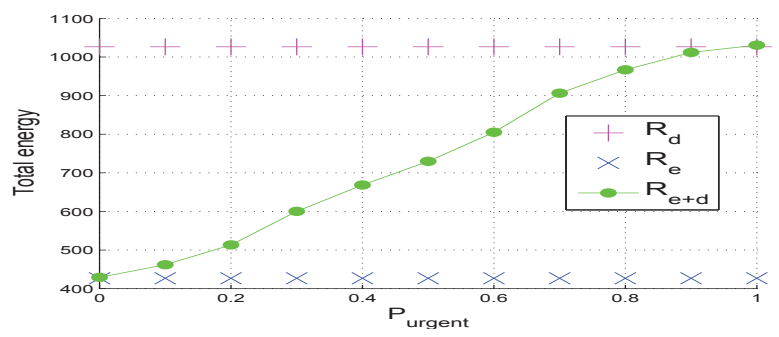

Fig. 5. Total consumed energy with varying purgent.

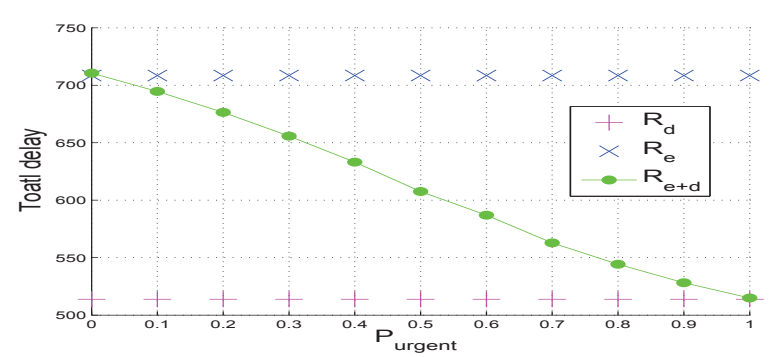

Fig. 6. Total end-to-end delay with varying $p_{\text {urgent }}$.

\section{Simulation RESUltS}

To validate our results, in a more realistic setting we build an event driven simulator in Matlab which simulates the different strategies. We consider a continuous monitoring application in which data are generated periodically at a predefined frequency $P$. In our simulation, we assume an underlying Low Power Listening $L P L$ link layer [12], [13]. We denote the average energy consumption when a packet is transmitted successfully, when the packet transmission failed, and when the packet is received for each radio $i=1,2$ by $E_{r_{i}}^{s t x}, E_{r_{i}}^{f t x}$, and $E_{r_{i}}^{r x}$, respectively, as follows:

$$
\begin{gathered}
E_{r_{i}}^{r x}=(\text { sample } / 2+\text { delay }) \times I_{r_{i}}^{r x} \times V \\
E_{r_{i}}^{s t x}=\left(L P L / 2 \times I_{r_{i}}^{t x}+\text { delay } \times I_{r_{i}}^{r x}\right) \times V \\
E_{r_{i}}^{f t x}=L P L \times I_{r_{i}}^{t x} \times V
\end{gathered}
$$

Based on equations (12) and (13), the average energy consumed when transmitting a packet over a link with $P R R_{r_{i}}(l)$ is

$\mathrm{E}_{r_{i}}^{t x}(l)=E_{r_{i}}^{f t x} \times\left(\frac{1}{P R R_{r_{i}}(l)}-1\right)+E_{r_{i}}^{s t x}+E_{r_{i}}^{r x}, i=1,2(14)$

where $L P L$ is the low power listening interval, $I_{r_{i}}^{t x}$ is the radios current draw when transmitting, $I_{r_{i}}^{r x}$ is the radios current draw when receiving, $V$ is the voltage, sample is the time it takes for a node to check the channel for activity, and delay is a constant time in which the radio is kept on after reception or transmission.

The network parameters are presented in Table II. It is to be noted that the parameters of the radio reflects the characteristics of our hardware $O p a l$ [1] supporting two radios $r_{1}$ and $r_{2}$ for $R F 230$ [14] and $R F 212$ [15], respectively.

TABLE II

Simulation PARAMETERS.

\begin{tabular}{|l|l|l|}
\hline Parameter & Meaning & Value \\
\hline delay & Delay constant & $20 \mathrm{~ms}$ \\
\hline sample & Sampling check time & $50 \mathrm{~ms}$ \\
\hline$L P L$ & Low Power Interval & random in $[100,1000] \mathrm{ms}$ \\
\hline$I_{r_{1}}^{t x}$ & Current of transmission with $r_{1}$ & $24 \mathrm{~mA}$ \\
\hline$I_{r_{2}}^{t x}$ & Current of transmission with $r_{2}$ & $16 \mathrm{~mA}$ \\
\hline$I_{r_{1}}^{r x}$ & Current of reception with $r_{1}$ & $9 \mathrm{~mA}$ \\
\hline$I_{r_{2}}^{r x}$ & Current of reception with $r_{2}$ & $15 \mathrm{~mA}$ \\
\hline$V$ & Voltage & 3 volts \\
\hline$P$ & Packet transmission period & 10 seconds \\
\hline
\end{tabular}

The following results are simulated in a grid topology of $4 \times 4$ nodes (as shown in Fig. 7).

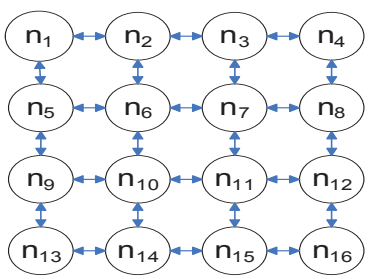

Fig. 7. Grid topology of 16 nodes.

In this scenario, we let $n_{16}$ be the packet source node and $n_{1}$ the destination node. The results are averaged over 100 simulation runs. Fig. 8 and Fig. 9 show the average energy consumed and the average end-to-end delay with varying $p_{\text {urgent }}$, respectively. As expected, we observe the same behavior for $R_{e+d}$ as in the analysis. Depending of the traffic that can occur in the network, $R_{e+d}$ achieves a trade-off of energy/delay. In fact, when $p_{\text {urgent }}=0, R_{e+d}$ follows the same behavior as $R_{e}$ to minimize the energy consumption, and when $p_{\text {urgent }}=1$, $R_{e+d}$ follows the same behavior as $R_{d}$ to minimize the endto-end delay. However, $R_{e+d}$ is in between the performance of both $R_{e}$ and $R_{d}$ when $0<p_{\text {urgent }}<1$.

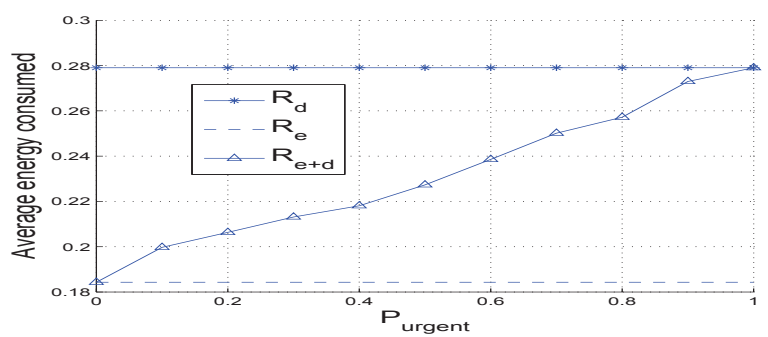

Fig. 8. Average energy consumed with varying $p_{\text {urgent }}, n_{16}$ is generating data.

Fig. 10 and Fig. 11 show the average of consumed energy and average end-to-end delay per number of generated packets, respectively, with simulation time. In this scenario, we set $p_{\text {urgent }}=0.5$ and only one node $\left(n_{16}\right)$ is periodicity generating data to be forwarded toward the $B S\left(n_{1}\right)$. The 


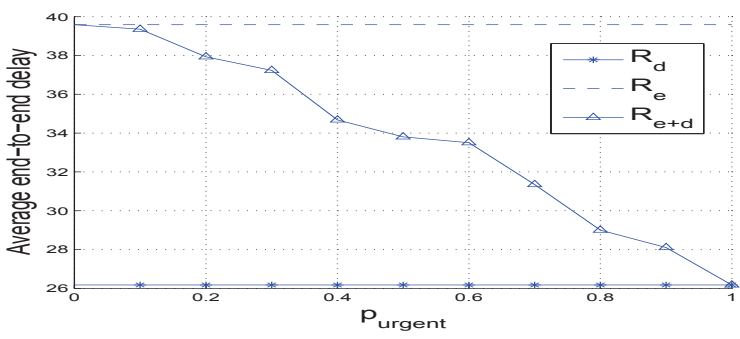

Fig. 9. Average end-to-end delay with varying $p_{\text {urgent }}, n_{16}$ is generating data.

results shown are averaged over 100 simulation runs and we used different seeds for the random $P R R$ generation in each simulation. The results confirm the analytical result, in which $R_{e}$ ensures the minimum energy consumption, while $R_{d}$ ensures a minimum end-to-end delay. However, $R_{e+d}$ adapts to the packet type. Indeed, depending on whether the generated packet is urgent or normal it is forwarded with either a minimum delay or a minimum energy, respectively. Note that, the increase of the energy consumption and the delay with simulation time are due to the increase of packet retransmissions over the time. For example, dividing the total energy consumed by the total transmitted packets gives a constant amount of energy consumption with time, which corresponds to 0.0141 Joules, 0.01009 Joules, and 0.006965 Joules for $R_{d}, R_{e+d}$, and $R_{e}$, respectively).

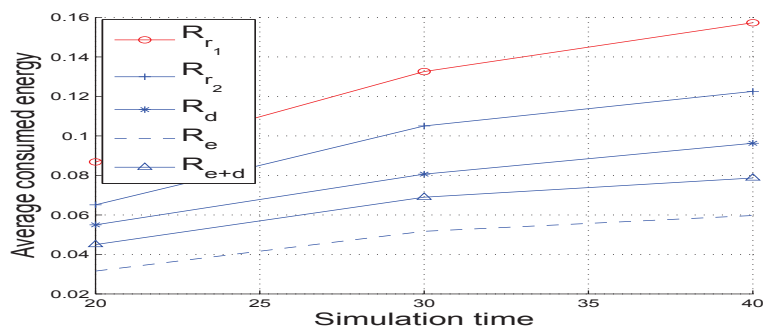

Fig. 10. Average consumed energy with simulation time, $n_{16}$ is generating data.

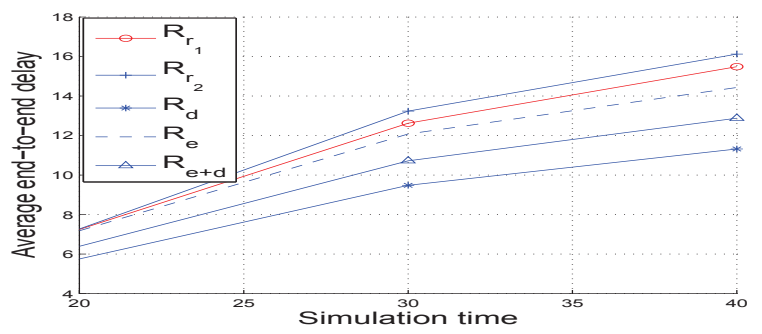

Fig. 11. Average end-to-end delay with simulation time, $n_{16}$ is generating data.

Similar results are obtained for different topologies and scenarios. They are omitted due to space limitation.

\section{CONCLUSiOn AND FUtURE WORK}

In this paper, we investigated the energy and delay interdependencies resulting from typical link quality fluctuations. We proposed a routing metric in the $M I M O$ model that can adapt to the type of the traffic that may be generated in a network. We developed an analytical model for the energy and the delay to compare between the different schemes regarding the energy consumption and the end-to-end delay. We also built an event driven simulator for the different strategies that reflects the energy consumption of our real hardware nodes to validate the performance of the proposed scheme. Simulation and analytical results show that the $R_{e}$ and the $R_{d}$ protocols minimize energy and delay, respectively, while the proposed protocol $R_{e+d}$, depending of the traffic, makes a trade-off between minimizing energy and end-to-end delay. For future works, we aim at exploring the increase of the number of radios within a sensor node and the impact it has on both the delay and energy.

\section{REFERENCES}

[1] B. Kusy, C. Richter, W. Hu, M. Afanasyev, R. Jurdak, M. Brünig, D. Abbott, C. Huynh, and D. Ostry, "Radio diversity for reliable communication in WSNs," in Proceding of International Symposium on Information Processing in Sensor Networks, (IPSN'2011), 2011.

[2] S. Moad, M. Tranberg Hansen, R. Jurdak, B. Kusy, and N. Bouabdallah, "WETX: Weighted Expected Transmission for Diversity in Wireless Sensor Networks," in Proceedings of International Federation for Information Processing (IFIP'2011), Wireless Days.

[3] S. Moad et al., "Load Balancing Metric with Diversity for Energy Efficient Routing in Wireless Sensor Networks," in Proceedings of International Symposium on Intelligent Systems Techniques for Ad hoc and Wireless Sensor Networks (IST-AWSN'2011).

[4] A. Paulraj, R. Nabar, and D. Gore, Introduction to space-time wireless communications. Cambridge Univ Pr, 2003.

[5] R. Draves, J. Padhye, and B. Zill, "Routing in multi-radio, multi-hop wireless mesh networks," in Proceedings of the 10th annual international conference on Mobile computing and networking, 2004, pp. 114128.

[6] R. Ketcham and J. Frolik, "A low-complexity, compact antenna for mitigating frequency-selective fading," in 6th International Symposium on Information Processing in Sensor Networks. IPSN'2007, 2007, pp. 573-574.

77] J. Liang and Q. Liang, "Channel selection in virtual MIMO wireless sensor networks," IEEE Transactions on Vehicular Technology, vol. 58, no. 5, pp. 2249-2257, 2009.

[8] A. Woo, T. Tong, and D. Culler, "Taming the underlying challenges of reliable multihop routing in sensor networks," in Proceedings of the 1st international conference on Embedded networked sensor systems, 2003, pp. 14-27.

[9] K. Srinivasan and P. Levis, "Rssi is under appreciated," in Proceedings of the Third Workshop on Embedded Networked Sensors (EmNets), vol. 2006, 2006.

[10] R. Fonseca, O. Gnawali, K. Jamieson, S. Kim, P. Levis, and A. Woo, "The collection tree protocol (CTP)," TEP 123 Draft, 2006.

[11] O. Gnawali, R. Fonseca, K. Jamieson, D. Moss, and P. Levis, "Collection tree protocol," in Proceedings of the 7th ACM Conference on Embedded Networked Sensor Systems, 2009, pp. 1-14.

[12] J. Polastre, J. Hill, and D. Culler, "Versatile low power media access for wireless sensor networks," in Proceedings of the 2nd international conference on Embedded networked sensor systems, November, 2004, pp. 03-05.

[13] D. Moss, J. Hui, and K. Klues, "Low Power Listening," TinyOS Core Working Group, TEP, vol. 105, 2007.

[14] “Atmel. AT86RF230 data sheet.” http://www.atmel.com/dyn/resources/prod documents/doc5131.pdf.

[15] “Atmel. AT86RF212 data sheet.” http://www.atmel.com/dyn/resources/prod_ documents/doc8168.pdf. 